\title{
Evaluation of the effect of endoscopic partial middle- turbinectomy surgery on the quality of life of patients with chronic rhinosinusitis and nasal polyps*
}

\author{
Mojtaba Maleki Delarestaghi', Shahin Rajaeih', Fatemeh Dehgani \\ Firouzabadi ${ }^{2}$, Mina Jamali², Maryam Roomiani², Mohammad Dehghani \\ Firouzabadi², Faramarz Memari ${ }^{1}$, Hesam Jahandideh ${ }^{3}$
}

${ }^{1}$ Firoozgar Clinical Research Development Center, Firoozgar Hospital, Iran University of Medical Sciences, Tehran, Iran.

2ENT and Head \& Neck Research Center, The Five Senses Institute, Hazrat Rasoul Hospital, Iran University of Medical Sciences, Tehran, Iran

${ }^{3}$ Department of Otolaryngology-Head and Neck Surgery, Firoozgar Hospital, Iran University of Medical Sciences, Tehran, Iran
Rhinology 58: 3, 208 - 212, 2020

https://doi.org/10.4193/Rhin19.258

*Received for publication:

July 25, 2019

Accepted: November 18, 2019

\begin{abstract}
Background: Chronic rhinosinusitis (CRS) is a common chronic inflammatory disease. Endoscopic sinus surgery is recommended as a standard method when medical treatment fails. The effectiveness of various complementary surgical methods such as endoscopic partial middle-turbinectomy is controversial in the improvement of CRS symptoms in these patients. This study aimed to investigate the effect of endoscopic partial middle-turbinectomy on the quality of life (QOL) of patients with chronic rhinosinusitis and nasal polyps (CRSwNP) in Iran.
\end{abstract}

Method: Ninety patients with CRSwNP of grades 3 and 4 were randomly assigned to either an intervention (45 patients) or control group (45 patients). In the control group, endoscopic sinus surgery without middle turbinectomy was performed and in the intervention group, endoscopic partial middle-turbinectomy was performed in addition to endoscopic sinus surgery. To evaluate the outcomes, the SNOT-22 QOL questionnaire was used, and the results were analyzed using SPSS version 24.

Results: Most of the patients were male and had a mean age of 39 years. The mean SNOT-22 QOL questionnaire scores were $49.13 \pm 16.72$ and $52.51 \pm 16.95$ before surgery in the control and intervention groups respectively, which did not show any significant difference. In contrast, after endoscopic surgery these scores changed to $28.46 \pm 12.38$ and $11.13 \pm 5.55$ in the control and intervention groups, respectively< and there was a significant difference between both groups. Although there was a significant improvement in both groups, the patients in the intervention group experienced more improvement than the control group (41.4 $( \pm 16.46)$ vs 30.7 ( \pm 18.27$)$, respectively

Conclusion: According to this study, it appears that the use of endoscopic partial middle-turbinectomy in addition to endoscopic sinus surgery improves CRS symptoms and the QOL of patients compared with endoscopic sinus surgery alone.

Key words: chronic rhinosinusitis, nasal polyposis, middle turbinate, endoscopic surgery, quality of life

\section{Introduction}

Chronic rhinosinusitis (CRS) is a common chronic inflammatory disease of the nose and paranasal sinuses ${ }^{(1)}$. The prevalence of this disease is estimated to be around $15 \%$ worldwide ${ }^{(2)}$ and is also associated with nasal polyps in $30 \%$ of patients ${ }^{(3)}$. Chronic rhinosinusitis with nasal polyps (CRSwNP) is a subtype of CRS that affects approximately $4 \%$ of the general population ${ }^{(4)}$.
About $20 \%$ of all patients referred to immunology and allergy clinics are diagnosed with CRSwNP ${ }^{(5)}$. Nasal congestion, rhinorrhea, insomnia, headache, and posterior nasal drip are the most common symptoms of CRSwNP that affect the daily activities of patients ${ }^{(6,7)}$. In addition, a high recurrence rate and multiple referrals to allergy clinics are consequences that not only place a financial burden on both the patient and society but also 
disrupt a patient's social and individual functions ${ }^{(8,9)}$ and reduce their quality of life (QOL).

QOL is defined as an individual's mental evaluation of the positive and negative aspects of their life ${ }^{(10)}$. Health-related QOL is a QOL evaluation component that evaluates a patient's perception of their health status in different areas. CRS symptoms such as insomnia, fatigue and olfactory dysfunction may change a patient's functional, emotional and social states and lead to a decrease in their overall QOL ${ }^{(10)}$. Previous studies have determined a $74 \%-94 \%$ reduction in the QOL of CRS patients ${ }^{(10,11)}$. Although medical therapy is the initial treatment for rhinosinusitis, surgical treatment is considered in relapsing cases or in cases with resistance to primary medical therapy ${ }^{(12)}$. Recently, sinus surgery has begun to involve endoscopic procedures as standard because of the advances in this technique ${ }^{(6,13)}$. In addition to standard endoscopic sinus surgery, some studies have shown positive effects, such as decreasing the recurrence rate of polyps and fewer synechiae when combining endoscopic partial middle turbinectomy with endoscopic sinus surgery ${ }^{(3,14,15)}$. However, some controversial ideas still remain concerning the beneficial effect of keeping the middle turbinate as a landmark in modifying surgeries as well as preventing frontal sinusitis ${ }^{(16)}$. Evaluating the QOL in patients with CRSwNP is an important indicator to measure not only the severity of the disease but also the effectiveness of each type of treatment in these patients. Recently, assessment of the beneficial effects of therapeutic procedures in chronic diseases including CRSWNP has changed from patient-reported outcomes to the evaluation of objective mental symptoms using tools such as QOL index ${ }^{(6)}$. QOL can be used as a clinical assessment of health in chronic diseases ${ }^{(11)}$. The effectiveness of endoscopic partial middle turbinectomy in CRSwNP patients on improving QOL was emphasized in previous studies ${ }^{(10,17,18)}$. However, evidence of its beneficial effects is conflicting and it seems essential to evaluate the QOL of patients in individual societies because different cultures, beliefs, and ethnicities have an impact on perceptions of QOL.

\section{Materials and methods}

\section{Patients}

This single-center, parallel-group, blinded, randomized clinical trial study was performed on 90 patients referred to Firoozgar hospital from 2017 to 2019 for CRSwNP. The inclusion criteria was a diagnosis of CRS based on Fokkens et al. criteria ${ }^{(19)}$, which consists of the presence of two or more symptoms that one of the symptoms must be one of these either nasal blockage, obstruction, congestion or nasal discharge, ( \pm facial pain or pressure, \pm reduction or loss of smell) for $\geq 12$ weeks. Additionally, the diagnosis of CRS was confirmed according to a nasal endoscopy and paranasal sinus CT scan. Moreover, patients with either grade 3 or 4 nasal polyps based on Meltzer et al. criteria ${ }^{(20)}$ were included. Exclusion criteria were having a history of previous endoscopic sinus surgery, secondary CRS due to fungal infections, vasculitis, mucocele, or anatomical deformities, or having a history of smoking, GERD, COPD, or immune suppression. Then, the patients were randomly divided into two 45 patient groups including an intervention group and a control group. Classic endoscopic sinus surgery was performed in both the control and intervention groups, and endoscopic partial middle turbinectomy was also performed in the intervention group. This project was granted ethical approval from Iran University of Medical Sciences ethics committee (IR.IUMS.FMD.REC No. 1396.9411369004). The informed consent letter was completed by all participants prior to the study.

\section{Surgical technique}

All patients underwent classical endoscopic sinus surgery as an uncinectomy, antrostomy, anterior and posterior ethmoidectomy, frontal sinusotomy and sphenoidotomy. Surgeons were blinded to the middle turbinate treatment method until the end of surgery when the treatment technique was revealed and performed. Endoscopic partial middle-turbinectomy was performed in 45 patients randomly assigned to the intervention group. Turbinate scissors were used to remove the anteroinferior part of the middle turbinate, while the upper part was left as a landmark. The posterior part of the middle turbinate, which is a landmark for sphenoidotomy was also protected. The rest of the patients were considered as a control group. A trans-septal 4-0 Vicryl suture was used to hold the turbinate in position in two patients in the control group due to its destabilization.

At the end of the surgery, nasal packing was placed in each middle meatus cavity for 3 days and oral cephalexin (500 mg, QID) was administered for 7 days and normal saline irrigation was recommended.

At the 2-week follow-up, endoscopic sinonasal cavity debridement was performed and fluticasone nasal spray (50 mcg/ spray) therapy was prescribed twice daily for both groups.

Pre-operative and post-operative outcome evaluation The Sino-Nasal Outcome Test version 22 (SNOT-22) questionnaire was completed preoperatively and 1 year after surgery ${ }^{(21)}$ to evaluate the QOL of patients. This questionnaire contains 22 questions about rhinosinusitis symptoms and is used as a specific QOL assessment for evaluating patients with CRSwNP. Each question is scored from zero (no problem) to five (the problem is as bad as possible) with final scores in the range of zero to 110 . Higher scores mean a lower QOL. The validity and reliability of the Persian version of the questionnaire were assessed in a previous study ${ }^{(22)}$. In addition, there was no significant difference between the two groups regarding adherence to medications during the follow-up period. Sinonasal endoscopy was performed as part of the routine post-operative care, and was carried out at each visit by a different blinded researcher after the SNOT- 
Figure1. Flow diagram of the progress through the phases of a parallelrandomized trial of two groups.

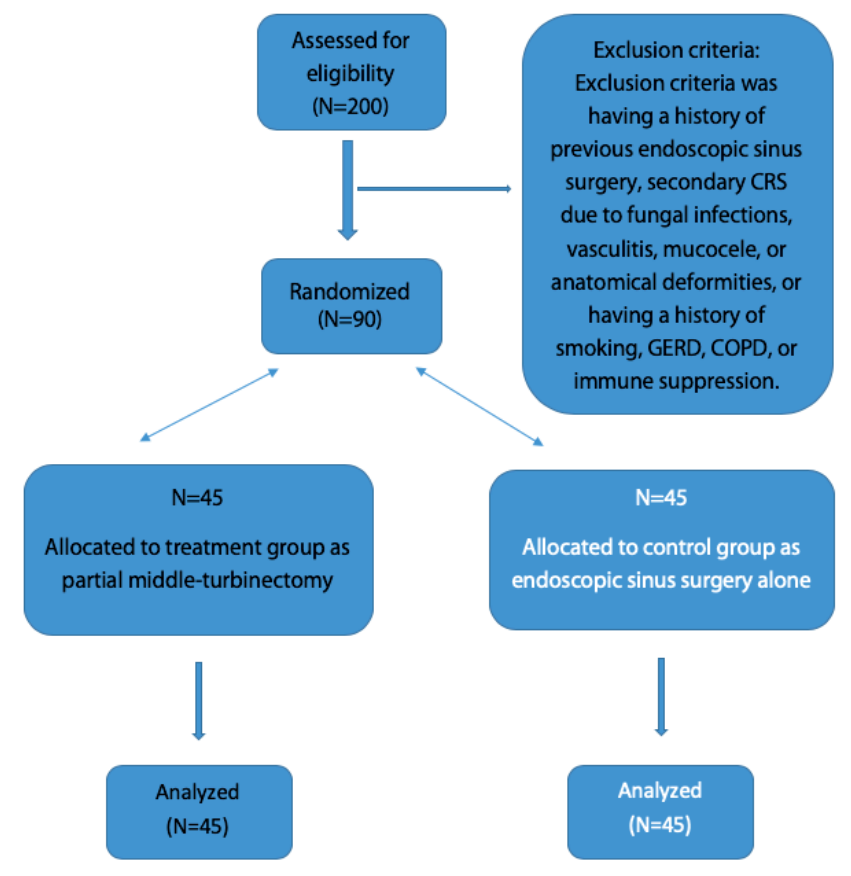

22 questionnaire was completed.

\section{Statistical analysis}

All statistical analyses were conducted using SPSS version 24 (IBM Corp. NY, USA). Statistical analysis of continuous variables with a normal distribution such as age and QOL evaluation were expressed as mean \pm standard deviation, and categorical variables such as sex and history of asthma and aspirin sensitivity were defined as proportions or ratios. Baseline characteristics of the study participants were compared using independent t-test, Mann-Whitney, or chi square tests where appropriate. Wilcoxon and Mann-Whitney tests were also used to evaluate preoperative and postoperative quantitative data. P-values under 0.05 were considered statistically significant.

\section{Results}

Ninety patients with CRSwNP were randomly divided into 2 groups: the intervention group (45 patients) and the control group (45 patients) (Figure 1). Sixty per cent of the patients were male with a mean age of 39 years old. Past medical history of asthma and aspirin sensitivity was collected. As shown in Table 1 , there was no significant difference between the two groups in aspirin sensitivity and having a history of asthma ( $p$-values=1.000). The effectiveness of endoscopic partial middle turbinectomy between the two groups is also shown in Table 2. The preoperative mean $\mathrm{QOL}$ scores in the intervention group and control group were $49.13 \pm 16.72$ and $52.51 \pm 16.95$ respectively, which was not significantly different ( $p$-value $=0.305$ ). However,
Table 1. Basic characteristic between both groups.

\begin{tabular}{|c|c|c|c|}
\hline Variables & $\begin{array}{l}\text { Intervention } \\
\text { group ( } N=45)\end{array}$ & $\begin{array}{l}\text { Control group } \\
(\mathrm{N}=45)\end{array}$ & p-value \\
\hline Age $^{a}$ & $39.73 \pm 14.05$ & $39.47 \pm 12.61$ & 0.840 \\
\hline Sex (Female /Male) ${ }^{\mathrm{b}}$ & $19 / 26$ & $17 / 28$ & 0.830 \\
\hline History of asthma ${ }^{b}$ & $6(13.3 \%)$ & $5(11.1 \%)$ & 1.000 \\
\hline Aspirin sensitivity ${ }^{b}$ & $8(17.8 \%)$ & $7(15.6 \%)$ & 1.000 \\
\hline
\end{tabular}

a according to Mann-Whitney test, ${ }^{b}$ according to Fisher's exact test.

Table 2. Comparison SNOT-22 scores between both groups.

\begin{tabular}{|c|c|c|c|c|}
\hline Outcomes & Group & $\begin{array}{c}\text { Pre- } \\
\text { operation }\end{array}$ & $\begin{array}{c}\text { One-year } \\
\text { post- } \\
\text { operation }\end{array}$ & p-value ${ }^{a}$ \\
\hline \multirow{3}{*}{$\mathrm{QOL}$} & $\begin{array}{l}\text { control } \\
\text { group }\end{array}$ & $49.13 \pm 16.72$ & $28.46 \pm 12.38$ & $<0.001$ \\
\hline & $\begin{array}{l}\text { intervention } \\
\text { group }\end{array}$ & $52.51 \pm 16.95$ & $11.13 \pm 5.55$ & $<0.001$ \\
\hline & P-value ${ }^{b}$ & 0.305 & 0.002 & \\
\hline
\end{tabular}

a according to Wilcoxon test, ${ }^{\mathrm{b}}$ according to Mann-Whitney exact test.
1 year after surgery, the mean SNOT-22 index scores were different in the intervention group and control group: $11.13 \pm 5.55$ and $28.46 \pm 12.38$, respectively ( $p$-value $=0.002$ ). Comparing the mean QOL scores showed that there was a significant difference between the scores obtained before surgery and 1 year after surgery in the intervention $(52.51 \pm 16.95$ to $11.13 \pm 5.55$, respectively, $\mathrm{p}$-value $<0.001)$, and the control groups $(49.13 \pm 16.72$ to $28.46 \pm 12.38$, respectively, $p$-value $<0.001)$. However, patients in the intervention group had a mean SNOT-22 score improvement of $41.4( \pm 16.46)$ compared with $30.7( \pm 18.27)$ in the control group. Despite significant improvement in both groups, the intervention group patients experienced more improvement than the control group ( $p$-value $=0.004$, independent $t$-test was performed).

\section{Discussion}

Surgery is one of the treatment methods for CRSwNP and is performed using different techniques ${ }^{(4)}$. Choosing an appropriate therapeutic approach for a disease is related to how effective it is in controlling the outcomes of the disease. In regard to the costs of CRSwNP for society and patients, a reliable guide to determine the effectiveness of therapeutic interventions in chronic diseases is the QOL index which can help when choosing an effective treatment ${ }^{(11)}$. Therefore, we aimed to evaluate the effect of endoscopic partial middle-turbinectomy surgery in patients with CRSwNP compared with the effect of endoscopic sinus surgery without partial middle turbinectomy. Based on the SNOT-22 scores evaluated in the study, the difference in QOL 
before and 1 year after surgery in patients with CRSwNP with or without endoscopic partial middle-turbinectomy showed that the treatment group had a better QOL improvement.

A common problem in CRSwNP is nasal obstruction that leads to nasal congestion, rhinorrhea, anosmia, headache, respiratory problems and posterior nasal drip ${ }^{(23)}$. Endoscopic partial middleturbinectomy can remove this obstruction, and so it plays an important role in improving symptoms, which in turn improves the QOL of patients. A comprehensive review study on the effectiveness of endoscopic sinus surgery techniques showed that these methods not only improve treatment outcomes and patients' symptoms, but also reduce the recurrence of polyps, which leads to further improvement in a patient's condition ${ }^{(24)}$. As with the results of our study, Marchioni et al. in 2008 showed that adding endoscopic partial middle-turbinectomy to endoscopic sinus surgery reduces CRS symptoms, which leads to a lower recurrence rate. A higher $\mathrm{QOL}$ index was also reported after 3 years of follow-up in the endoscopic partial middle-turbinectomy patients ${ }^{(25)}$. Similarly, Hoodon and colleagues investigated the effectiveness of this surgical method in a two-group study by examining symptoms 1,3 , and 6 months after surgery. The results showed that even though the amount of crust in the turbinectomy group was greater than in the control group in the first month, at the postoperative 6-month follow-up visit, patients in the intervention group had a significant reduction in all symptoms ${ }^{(13)}$. Therefore, diminishing symptoms, reducing complications and improving QOL in patients with CRSwNP can justify the effectiveness of this surgical procedure.

However, Soler and colleagues showed that in CRS patients treated surgically with and without partial middle-turbinectomy, there was no significant difference between the QOL score of patients before and after surgery based on the SF-36 QOL questionnaire ${ }^{(26)}$. This disagreement can be related to the difference in the measurement tools and supports the use of a specific QOL tool based on the nature of the disease. General QOL measures such as the SF-36 are always used to compare the effects of different illnesses on health, while specific tools such as the SNOT-22 questionnaire are used to compare specific features of total health.

Saedi et al evaluated the most optimal management of the middle turbinate in an epidemiological study on 90 patients with nasal polypsin 2014. They used three different surgical procedures: middle-turbinectomy by radiofrequency, partial resection of the middle turbinate, and medialization of the middle turbinate. Their results showed that all three treatment methods could improve symptoms and there was no statistically significant difference in patients' $\mathrm{QOL}$ in the three groups ${ }^{(27)}$.

There are also studies that emphasize maintaining the turbinate structure. Some studies have reported complications such as atrophic rhinitis, iatrogenic frontal sinusitis, anosmia and empty nose syndrome, which are not consistent with the results of our study ${ }^{(28,29)}$. However, the removal of the middle turbinate is still controversial based on some studies ${ }^{(13,30)}$.

The strength of this surgical procedure should be considered due to the decrease in the recurrence rate of polyps and its impact on improving the QOL of patients, as well as its effectiveness in reducing healthcare costs, which has been reported in numerous studies. Based on this, we believe that the present study could lead to further research in this field in Iran.

There are some limitations in this study. First, we tried to consider some potential confounders as exclusion criteria, but it would be better for future studies to study the smoker population due to the higher risk of scarring and crusting in this population. Second, as any difference between these two grades of nasal polyps(type 3 and 4) between both groups(intervention and control groups) could lead to a study bias, it is better to analyze the percentage of each grade between two groups in the future studies. Although we subjectively assessed patients using a SNOT-22 questionnaire post-operatively, objective methods of outcome evaluation by endoscopy and/or imaging will be of more value.

\section{Conclusion}

Finally, the results of this study support the effectiveness of endoscopic partial middle-turbinectomy as a treatment for CRSwNP in improving patients' symptoms and QOL. Further clinical trials are necessary to determine the effectiveness of this surgical procedure.

\section{Authorship contribution}

MMD, SHR, HJ: Organizing and supervising the course of the article and taking the responsibility; MJ, MR, MDF: Taking responsibility in patient follow-up, data management and reporting; MMD, FDF, FM, MDF: Taking responsibility in the construction of the body of the manuscript; MMD, FDF, HJ, FM: Reviewing the article before submission not only for spelling and grammar but also for its intellectual content.

\section{Conflict of interest}

None.

\section{References}

1. Paul Flint, Bruce Haughey, Valerie Lund, John Niparko, K. Robbins, J. Regan Thomas, et al. Cummings Otolaryngology 2014.

2. Madani SA, Hashemi SA, Fazli M, Esfandiar
K. Bacteriology in Patients With Chronic rhinosinusitis in North of Iran (Sari). Jundishapur J Microbiol. 2013;6(8).

3. Bachert C, Zhang L, Gevaert P. Current and future treatment options for adult chronic rhinosinusitis: Focus on nasal polyposis. J Allergy Clin immunol. 2015;136(6):1431-40.

4. Amali A, Bidar Z, Rahavi-Ezabadi S, Mikaniki N, Sadrehosseini SM. Polypoid change of middle turbinate is associated 
to an increased risk of polyp recurrence after surgery in patients with chronic rhinosinusitis with nasal polyps. Eur Arch Otorhinolaryngol. 2018 Aug;275(8):20212025

5. Hooshang Gerami, Rahmatollah Banan Dr, Shadman Nemati, Ali Akbar Fallahi, Al Mojtahedi, Soheil Soltanipour, et al. The Relative Frequency of Allergic Funga Rhinosinusitis in Patients with Nasal Polyposis in Rasht City, Iran. Qom Univ Med Sci J. 2017;10(12)

6. Fereshteh Esmatinia, bakhshaei $M$ Recurrent sinonasal polyposis after the endoscopic sinus surgery. Rev Clin Med. 2014; 1 (2):86-92

7. Marambaia PP, Lima MG, Guimaraes MB Gomes AM, Marambaia MP, Santos OMD, et al. Can we use the questionnaire SNOT-22 as a predictor for the indication of surgical treatment in chronic rhinosinusitis? Braz Otorhinolaryngol. 2017;83(4):451-6.

8. DeConde AS, Soler ZM. Chronic rhinosinusitis: Epidemiology and burden of disease. AmJ Rhinol Allergy. 2016;30(2):134-9.

9. Noon E, Hopkins C. Review article: outcomes in endoscopic sinus surgery. BMC Ear Nose Throat Disord. 2016;16(1):9

10. Rudmik L, Smith TL. Quality of life in patients with chronic rhinosinusitis. Curr Allergy Asthma Rep. 2011;11(3):247-52.

11. Nyaiteera $V$, Nakku D, Nakasagga E, Llovet E, Kakande E, Nakalema G, et al. The burden of chronic rhinosinusitis and its effect on quality of life among patients re-attending an otolaryngology clinic in south western Uganda. BMC Ear Nose Throat Disord. 2018;18(1):10

12. Meymane Jahromi A, Shahabi Pour A. The Epidemiological and Clinical Aspects of Nasal Polyps that Require Surgery. Iran J Otorhinolaryngol. 2012;24(67):75-8.

13. Hudon MA, Wright ED, Fortin-Pellerin E, Bussieres M. Resection versus preservation of the middle turbinate in surgery for chronic rhinosinusitis with nasal polyposis: a randomized controlled trial. J Otolaryngol Head Neck Surg. 2018;47(1):67.

14. Jang DW, Lachanas VA, Segel J, Kountakis SE. Budesonide nasal irrigations in the postoperative management of chronic rhinosinusitis. Int Forum Allergy Rhinol.
2013;3(9):708-11.

15. Wu AW, Ting JY, Platt MP, Tierney HT, Metson R. Factors affecting time to revision sinus surgery for nasal polyps: a 25-year experience. Laryngoscope. 2014;124(1):29-33.

16. Nurse LA, Duncavage JA. Surgery of the inferior and middle turbinates. Otolaryngol Clin North Am. 2009;42(2):295-309, ix.

17. Lin HP, Li N, Xu T, Jiang $Y, Y u L G$. [The impacts of endoscopic sinus surgery with middle turbinate and superior turbinate resection on quality of life and olfactory function in patients with chronic rhinosinusitis with nasal polyps]. Lin chuang er bi yan hou tou jing wai ke za zhi = J Clin Otorhinolaryngol, Head Neck Surg. 2016:30(16):1283-6.

18. Alt JA, DeConde AS, Mace JC, Steele TO, Orlandi RR, Smith TL. Quality of Life in Patients With Chronic Rhinosinusitis and Sleep Dysfunction Undergoing Endoscopic Sinus Surgery: A Pilot Investigation of Comorbid Obstructive Sleep Apnea. JAMA Otolaryngol Head Neck Surg. 2015;141(10):873-81.

19. Fokkens WJ, Lund VJ, Mullol J, Bachert C, Alobid I, Baroody F, et al. EPOS 2012: European position paper on rhinosinusitis and nasal polyps 2012. A summary for otorhinolaryngologists. Rhinology. 2012;50(1):1-12

20. Meltzer EO, Hamilos DL, Hadley JA Lanza DC, Marple BF, Nicklas RA, et al. Rhinosinusitis: developing guidance for clinical trials. J Allergy Clin Immunol. 2006;118(5 Suppl):S17-61.

21. Buckland JR, Thomas S, Harries PG. Can the Sino-nasal Outcome Test (SNOT-22) be used as a reliable outcome measure for successful septal surgery? Clin Otolaryngol Allied Sci. 2003;28(1):43-7.

22. Jalessi M, Farhadi M Kamrava SK Amintehran E, Asghari A, Rezaei Hemami M, et al. The reliability and validity of the persian version of sinonasal outcome test 22 (snot 22) questionnaires. Iran Red Crescent Med J. 2013;15(5):404-8.

23. Wu D, Bleier BS, Wei Y. Temporary olfactory improvement in chronic rhinosinusitis with nasal polyps after treatment. Eur Arch Otorhinolaryngol. 2018;275(9):2193-202.

24. Weber RK, Hosemann W. Comprehensive review on endonasal endoscopic sinus surgery. Curr Top Otorhinolaryngol Head Neck Surg. 2015;14:Doc08.

25. Marchioni D, Alicandri-Ciufelli M, Mattioli F, Marchetti A, Jovic G, Massone F, et al. Middle turbinate preservation versus middle turbinate resection in endoscopic surgical treatment of nasal polyposis. Acta otolaryngologica. 2008;128(9):1019-26.

26. Soler ZM, Wittenberg E, Schlosser RJ, Mace JC, Smith TL. Health state utility values in patients undergoing endoscopic sinus surgery. Laryngoscope. 2011;121(12):2672-8.

27. Saedi B, Amali A, Alizadeh N, Hwang $P$, Meisami AP. The effect of radiofrequency turbinoplasty vs two other methods in the management of polypoid changes of the middle turbinate: a randomized trial. Int Forum Allergy Rhinol. 2014;4(12):1030-4.

28. Gulati SP, Wadhera R, Kumar A, Gupta A, Garg A, Ghai A. Comparative evaluation of middle meatus antrostomy with or without partial middle turbinectomy. Indian J Otolaryngol Head Neck Surg. 2010;62(4):400-2.

29. Scheithauer MO. Surgery of the turbinates and "empty nose" syndrome. GMS Curr Top Otorhinolaryngol Head Neck Surg. 2010;9:Doc03.

30. Yasan H. Significance of nasal polyps in chronic rhinosinusitis: symptoms and surgical outcomes. Laryngoscope. 2005; 115(7):1320; author reply 1

Hesam Jahandideh

Department of Otolaryngology

Head and Neck Surgery

Firoozgar Hospital

Iran University of Medical Sciences

Tehran

Iran

E-mail: h.jahandideh@gmail.com

ORCID ID: 0000-0002-3084-1439 